7. Al-Hazmi H. Role of duration of catheterization and length of hospital stay on the rate of catheter-related hospital-acquired urinary tract infections. Res Rep Urol 2015;7:41-77.

8. Chopra V, Govidan S, Kuhn L, et al. Do clinicians know which of their patients have central venous catheters? A multicenter observational study. Ann Intern Med 2014;161:562-567.
9. Carrouget J, Legeay C, Poirier A, Azzouzi A-R, Zahar J-R, Bigot P. Enquête de prévalence sur le sondage vésical dans un centre hospitalo-universitaire. Progrès en Urologie 2017;27(5):305-311.

10. Baek H, Munsu C, Seok K, et al. Analysis of length of hospital stay using electronic health records: a statistical and data mining approach. PloS One 2018;13(4);e0195901.

\title{
Intrapatient transfer of an uncommon carbapenemase in Nebraska
}

\author{
Alyssa K. W. Maclean BS ${ }^{1,2}$ (1), Stacey Morrow MS, MT(ASCP) $)^{1,2}$, Anum Abbas MD ${ }^{3}$ (1), Renuga Vivekanandan $\mathrm{MD}^{3}$ (1), \\ Stephen Cavalieri $\mathrm{PhD}^{1,2,4,5}$ and Nancy D. Hanson $\mathrm{PhD}^{1,2}$ (1) \\ ${ }^{1}$ Department of Medical Microbiology and Immunology, Creighton University School of Medicine, Omaha, Nebraska, ${ }^{2}$ Center for Research in Anti-Infectives and \\ Biotechnology, Creighton University School of Medicine, Omaha, Nebraska, ${ }^{3}$ Division of Infectious Diseases, Creighton University School of Medicine, Omaha, \\ Nebraska, ${ }^{4}$ Department of Pathology, Creighton University School of Medicine, Omaha, Nebraska and ${ }^{5} \mathrm{CHI}$ Health Microbiology Laboratory, Omaha, Nebraska
}

To the Editor-Carbapenem-resistant Enterobacterales are a major medical concern, especially during the coronavirus disease 2019 (COVID-19) pandemic because bacterial superinfections in severe acute respiratory coronavirus virus 2 (SARS-CoV-2)infected patients have led to poor outcomes. ${ }^{1}$ Enterobacterales can emerge resistant to carbapenems through multiple mechanisms including the acquisition of carbapenemase genes on mobile genetic elements such as plasmids. These mobile genetic elements are a major concern due to the potential spread of carbapenem resistance and other resistance elements between multiple bacterial species. ${ }^{2,3}$ Carbapenemase-producing Enterobacterales (CRE) are found worldwide and are widespread in the United States, including in the state of Nebraska. ${ }^{4}$ Although KPC is the most commonly identified carbapenemase in the United States, New Delhi metallo$\beta$-lactamase (NDM) carbapenemases have been reported since $2010^{5}$ and have infected patients with and without history of international travel. ${ }^{3}$ In Nebraska, routine screening for carbapenem resistance has been recommended since 2017. Phenotypic or genotypic confirmation of carbapenemase production is performed by the Nebraska Public Health Lab. ${ }^{6}$ We describe the first documented case of infection with an NDM-7-producing Enterobacterales in the state of Nebraska. Furthermore, this case indicates the potential for plasmid spread to multiple species within a single patient.

Bacterial identification and antimicrobial susceptibility testing were performed using MicroScan Walkaway (Beckman Coulter, Brea, CA). Phenotypic determination of carbapenemase production for CRE was performed using the modified carbapenem inactivation method (mCIM) as described by the Clinical and Laboratory Standards Institute (CLSI). ${ }^{7}$ According to the Nebraska Department of Health and Human Services protocol, carbapenem-resistant isolates were sent to the Nebraska Public Health Laboratory for genotypic determination of carbapenemase production using Xpert Carba-R (Cepheid, Sunnyvale, CA). Following the initial identification and positive mCIM test, the isolate was sent to our laboratory for further evaluation. Confirmation of the presence of the NDM gene as well as the absence of other

Author for correspondence: Nancy D. Hanson, E-mail: ndhanson@creighton.edu

Cite this article: Maclean AKW, et al. (2022). Intrapatient transfer of an uncommon carbapenemase in Nebraska. Infection Control \& Hospital Epidemiology, 43: 535-536, https://doi.org/10.1017/ice.2021.33 carbapenemase genes was determined using the Streck ARM-D $\beta$-lactamase identification kit according to manufacturer's instructions. The NDM allele was further identified by Sanger sequencing. Plasmid carriage of NDM-7 was confirmed by Southern blotting using NDM-specific probes. ${ }^{8}$

A middle-aged African-American male presented in the emergency room with a left-foot ulcer associated with poorly controlled diabetes melitus type 2 (Fig. 1A). The patient had a long-standing issue with ulcers in his feet and had been followed by a podiatrist for several years. Patient history was significant for previous foot ulcers positive for methicillin-susceptible Staphylococcus aureus and recent travel to the Middle East for work. The diabetic foot ulcer was initially treated in the Middle East with surgical debridement and amputation of the second toe. The patient was prescribed amoxicillin-clavulanate and discharged. While traveling in Nebraska, the patient presented in the emergency room for a wound check. Upon presentation, the left foot had several deep ulcers in stage III and IV that appeared to have good granulation tissue, and no tenderness or purulence was noted. Laboratory tests revealed a normal white blood cell count of $11.810^{3} / \mathrm{mm}^{3}$; elevated creatinine at $2.61 \mathrm{mg} / \mathrm{dL}$ (baseline $2.0 \mathrm{mg} / \mathrm{dL}$ ); C-reactive protein (CRP) of $137 \mathrm{mg} / \mathrm{L}$; erythrocyte sedimentation rate $(E S R)>120 \mathrm{~mm} /$ hour. Cultures of the foot were obtained, and the patient was discharged with topical bacitracin. Upon consultation with the infectious disease physician several days later, further cultures were obtained, and the patient was started on oral levofloxacin.

Initial cultures were positive for carbapenem-resistant Enterobacter cloacae (Figure 1B). Follow-up cultures were positive for carbapenem-resistant Klebsiella pneumoniae and methicillinsusceptible Staphylococcus aureus (susceptibility not shown). For both Enterobacterales isolates, the mCIM test was positive. Realtime polymerase chain reaction (PCR) using the Streck ARM-D $\beta$-lactamase identification kit was positive for NDM in both the E. cloacae isolate (Entb 348) and the K. pneumoniae isolate (Kleb 407). Sanger sequencing of the NDM gene in both isolates identified the NDM-7 carbapenemase gene. Southern blots were performed to determine the location of the NDM-7 gene. Both strains carried a plasmid of the same size encoding NDM-7, suggesting likely conjugative transfer (data not shown). 


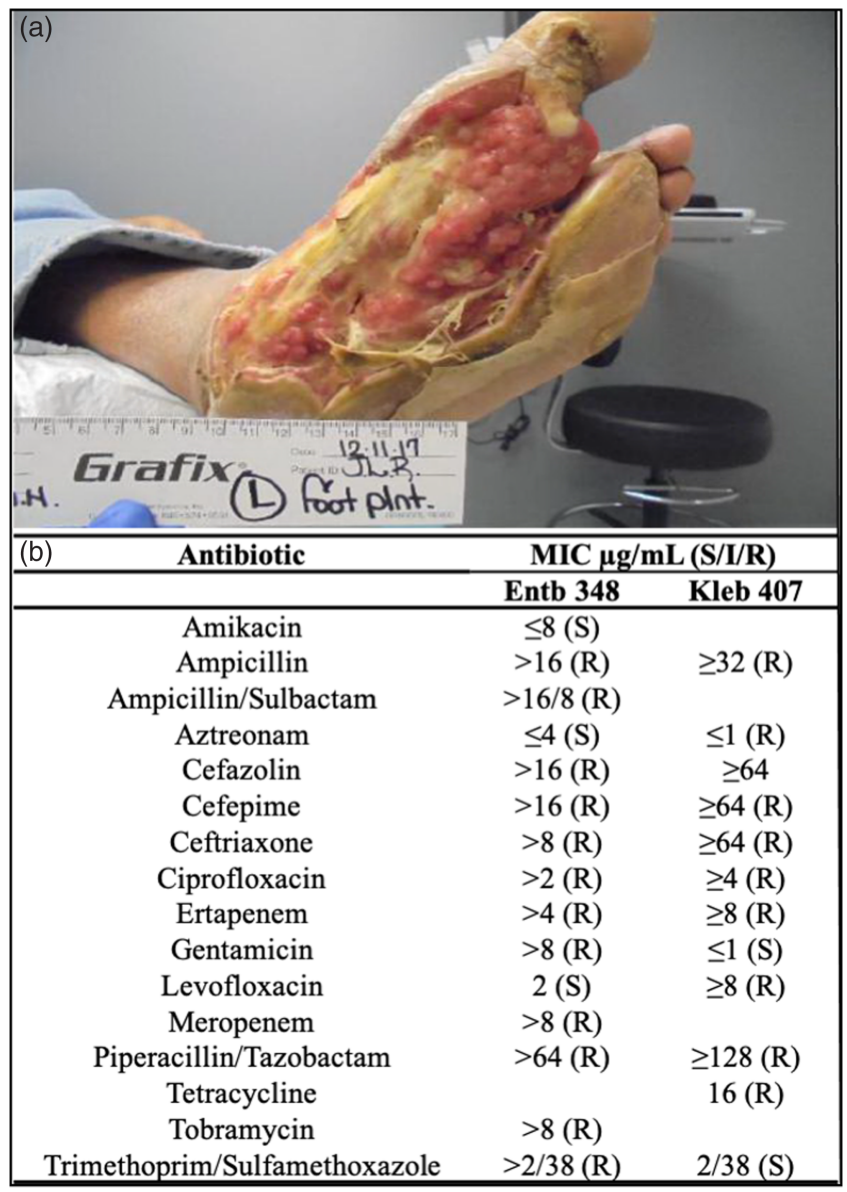

Fig. 1. Two NDM-7-producing Enterobacterales were isolated from a diabetic foot ulcer. (A) The left-plantar diabetic ulcer at the initial presentation. (B) Susceptibilities of the Enterobacter cloacae (Entb 348) and Klebsiella pneumoniae (Kleb 407) isolates indicate carbapenem resistance mechanisms.

The infectious disease physician elected to continue the levofloxacin because of clinical improvement despite apparent resistance in the K. pneumoniae. Tissue cultures obtained at wound care visits a few weeks later were positive for methicillin-resistant $S$. aureus, but no resistant gram-negative organisms were detected at that time. The patient was switched from levofloxacin to linezolid for the $S$. aureus and was scheduled for further debridement and flap. The patient had a split-thickness graft placed several weeks later over the left-foot ulcers, and the infections resolved.

NDM-7 was initially identified in Germany in 2012. This NDM variant is of particular concern due to greater hydrolytic activity against carbapenems than other NDM variants and high potential for horizontal gene transfer. ${ }^{9}$ To date, NDM-7 has only been identified in the United States in patients with a history of international travel. ${ }^{10}$ To our knowledge, this case represents the first identification of NDM-7-producing Enterobacterales in Nebraska. Although this patient's history is significant for international exposure, the incidence of NDM-producing Enterobacterales infection without international travel has been increasing. ${ }^{3}$ Thus, the identification of NDM $\beta$-lactamases is a cause for concern, regardless of patient history. The spread of the NDM-7 $\beta$-lactamase between E. cloacae and K. pneumoniae, as well as evidence of plasmid carriage of the gene, highlights the mobile nature of this resistance determinant. Swift action of the ID team and the outpatient setting can prevent transfer of the NDM-7 plasmid to other patients. Robust surveillance for these uncommon $\beta$-lactamases and appropriate precautions upon identification are necessary to ensure that their presence remains limited.

Acknowledgments. We thank the Creighton School of Medicine and the Department of Medical Microbiology and Immunology for supporting this project.

Financial support. No financial support was provided relevant to this article.

Conflicts of interest. All authors report no conflicts of interest relevant to this article.

\section{References}

1. Nori P, Szymczak W, Puius Y, et al. Emerging copathogens: New Delhi metallo-beta-lactamase-producing Enterobacterales infections in New York City COVID-19 patients. Int J Antimicrob Agents 2020;56:106179.

2. Prussing C, Snavely EA, Singh N, et al. Nanopore MinION sequencing reveals possible transfer of $b l a_{\mathrm{KPC}-2}$ plasmid across bacterial species in two healthcare facilities. Front Microbiol 2020;11:1-11.

3. van der Mee-Marquet N, Diene SM, Chopin F, Goudeau A, François P. Enigmatic occurrence of NDM-7 enzyme in the community. Int $J$ Antimicrob Agents 2016;47:505-507.

4. Facility guidance for control of carbapenem-resistant Enterobacteriaceae (CRE) November 2015 update. Centers for Disease Control and Prevention website. https://www.cdc.gov/hai/organisms/cre/cre-facilities.html. Published 2019. Accessed October 5, 2020.

5. Detection of Enterobacteriaceae isolates carrying metallo-beta-lactamaseUnited States, 2010. Morb Mortal Wkly Rep June 25, 2010;59:750.

6. Carbapenem-Resistant Enterobacteriaceae Investigation Guidelines. Nebraska Department of Health and Human Services website. http://dhhs. ne.gov/Pages/Antimicrobial-Resistance.aspx. Published 2017. Accessed October 5, 2020.

7. Weinstein MP, Lewis II JS, Bobenchik AM, et al. M100: Performance Standards for Antimicrobial Susceptibility Testing, 30th edition. Clinical Laboratory Standards Institute; 2020.

8. Sambrook J, Russel DW. Molecular Cloning, A Laboratory Manual, Volume 1, third edition. Cold Spring Harbor, NY: Cold Spring Harbor Laboratory Press; 2001.

9. Göttig S, Hamprecht AG, Christ S, Kempf VAJ, Wichelhaus TA. Detection of NDM-7 in Germany, a new variant of the New Delhi metallo-ß-lactamase with increased carbapenemase activity. J Antimicrob Chemother 2013;68: 1737-1740.

10. Lee CS, Vasoo S, Hu F, Patel R, Doi Y. Klebsiella pneumoniae ST147 coproducing NDM-7 carbapenemase and RmtF 16S rRNA methyltransferase in Minnesota. J Clin Microbiol 2014;52:4109-4110. 